\title{
A Four-Step Enzymatic Cascade for Efficient Production of L- Phenylglycine from Biobased L-Phenylalanine
}

\author{
Yuling Zhu ${ }^{[a]}$, Jifeng Yuan ${ }^{[a]^{*}}$
}

\begin{abstract}
Enantiopure amino acids are of particular interest in the agrochemical and pharmaceutical industries. Here, we reported a multi-enzyme cascade for efficient production of L-phenylglycine ( $L$ Phg) from biobased L-phenylalanine (L-Phe). We first attempted to engineer Escherichia coli for expressing L-amino acid deaminase (LAAD) from Proteus mirabilis, hydroxymandelate synthase (HmaS) from Amycolatopsis orientalis, (S)-mandelate dehydrogenase (SMDH) from Pseudomonas putida, the endogenous aminotransferase (AT) encoded by ilvE and L-glutamate dehydrogenase (GluDH) from E. coli. However, $10 \mathrm{mM}$ L-Phe only afforded the synthesis of $7.21 \pm 0.15 \mathrm{mM} \mathrm{L}$-Phg. The accumulation of benzoylformic acid suggested that the transamination step might be rate-limiting. We next used leucine dehydrogenase (LeuDH) from Bacillus cereus to bypass the use of L-glutamate as amine donor, and $40 \mathrm{mM} \mathrm{L}$-Phe gave $39.97 \pm 3.84 \mathrm{mM}(6.04 \pm 0.58 \mathrm{~g} / \mathrm{L}) \mathrm{L}-\mathrm{Phg}$, reaching $99.9 \%$ conversion. In summary, this work demonstrated a concise four-step enzymatic cascade for the L-Phg synthesis from biobased L-Phe, with a potential for future industrial applications.
\end{abstract}

Enantiopure amino acids are of particular interest in the agrochemical and pharmaceutical industries ${ }^{[1]}$. For example, Lphenylglycine (L-Phg) can be used for the synthesis of $\beta$-lactam antibiotics such as penicillin ${ }^{[2]}$, streptogramin $\mathrm{B}^{[3]}$, pristinamycin $I^{[4]}$ and virginiamycin $S{ }^{[5]}$. A number of chemical synthesis routes have been developed for the production of enantiopure amino acid ${ }^{[6]}$. For instance, the Strecker reaction is a common method for the industrial production of $\alpha$-amino acids ${ }^{[7]}$. However, chemical approaches typically use environmentally harmful toxic reagents and organic solvents, and the enantioselectivity of the product is relatively low, which are undesirable for sustainable production of enantiopure amino acids ${ }^{[8]}$.

In comparison to chemical approaches, biocatalytic processes are usually carried out under less harsh conditions such as ambient temperature and atmospheric pressure ${ }^{[9]}$. In addition, biocatalytic processes are considered as a suitable alternative for chiral chemical productions because of the excellent enantioselectivity ${ }^{[10]}$. Since amino acids such as Lphenylalanine (L-Phe) can be obtained by fermentation or from protein waste hydrolysates in large amounts and at a low cost ${ }^{[11]}$ they are considered to be cheap and renewable feedstocks for biomanufacturing applications. For instance, it was reported that the production of L-Phg from biobased L-Phe was achieved in the recombinant Escherichia coli containing eight reaction steps

[a] Yuling Zhu, Jifeng Yuan

State Key Laboratory of Cellular Stress Biology

School of Life Sciences, Xiamen University, Fujian 361102, PR China

E-mail: jfyuan@xmu.edu.cn

Supporting information for this article is given via a link at the end of the document. with 12 genes ${ }^{[12]}$. Under the optimal condition, $40 \mathrm{mM}$ L-Phe was converted to $34 \mathrm{mM}(5.1 \mathrm{~g} / \mathrm{L}) \mathrm{L}-\mathrm{Phg}$ after $24 \mathrm{~h}$ via the whole-cell biotransformation, reaching $\sim 85 \%$ conversion. In addition, de novo synthesis of L-Phg ${ }^{[14]}$ and in vitro enzyme catalysis ${ }^{[15]}$ have also been reported for the L-Phg production, the L-Phg yields were relatively low $(51.6 \mathrm{mg} / \mathrm{g} \mathrm{DCW}$ and 91.4 $\mathrm{mg} / \mathrm{L}$, respectively).

In this study, we sought to develop a more concise multi-enzyme cascade for synthesizing L-Phg from biobased L-Phe. In particular, our system comprises L-amino acid deaminase (LAAD, Uniprot ID: B2ZHY0) from Proteus mirabilis [13], hydroxymandelate synthase (HmaS, Uniprot ID: 052791) from Amycolatopsis orientalis [14], (S)-mandelate dehydrogenase (SMDH, Uniprot ID: P20932) from Pseudomonas putida ${ }^{[15]}$ and leucine dehydrogenase (LeuDH, Uniprot ID: P0A393) from Bacillus cereus ${ }^{[16]}$. The four-step enzymatic cascade could efficiently convert $40 \mathrm{mM} \mathrm{L}-\mathrm{Phe}$ to $39.97 \pm 3.84 \mathrm{mM}(6.04 \pm 0.58$ $\mathrm{g} / \mathrm{L}) \mathrm{L}$-Phg after $12 \mathrm{~h}$, with a conversion rate $>99.9 \%$. To the best of our knowledge, our work represents one of the most efficient biocatalytic routes for L-Phg synthesis from L-Phe ${ }^{[12]}$.

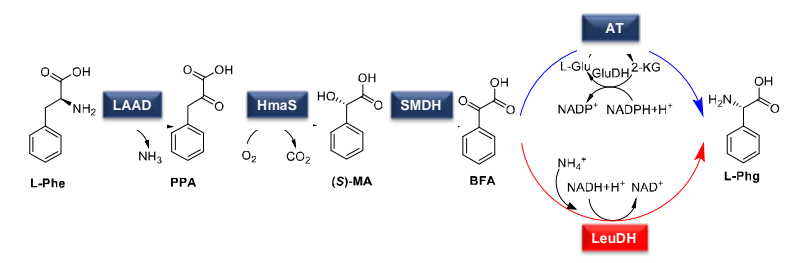

Scheme 1. Schematic diagram of multi-enzyme cascade toward L-Phg synthesis from biobased L-Phe. LAAD, L-amino acid deaminase from $P$. mirabilis (Uniprot ID: B2ZHY0); HmaS, hydroxymandelate synthase from $A$. orientalis (Uniprot ID: O52791); SMDH, (S)-mandelate dehydrogenase from $P$. putida (Uniprot ID: P20932); AT, aminotransferase encoded by ilvE from $E$. coli (Uniprot ID: P0AB80); GluDH, L-glutamate dehydrogenase from E. coli (Uniprot ID: P00370); LeuDH, leucine dehydrogenase from B. cereus (Uniprot ID: P0A393). L-Phe, L-phenylalanine; PPA, phenylpyruvate; (S)-MA, (S)mandelic acid; BFA, benzoylformic acid; L-Phg, L-phenylglycine; 2-KG, 2ketoglutarate; L-Glu, L-glutamate.

Recently, our group have demonstrated that LAAD from $P$. mirabilis and $\mathrm{HmaS}$ from $A$. orientalis together with $\mathrm{SMDH}$ from $P$. putida could effectively synthesize benzyl alcohol and benzylamine from L-Phe ${ }^{[17]}$. To enable L-Phg production from benzoylformic acid (BFA), we chose aminotransferase (AT, Uniprot ID: POAB80) encoded by ilvE and L-glutamate dehydrogenase (GluDH, Uniprot ID: P00370) from E. coli ${ }^{[12,18]}$. In brief, the multi-enzyme cascade comprises LAAD from $P$. mirabilis, HmaS from $A$. orientalis, SMDH from $P$. putida and AT/GluDH from E. coli (Scheme 1). As depicted in Figure 1a, the multi-enzyme cascade was recast into three modules: LAAD together with HmaS to convert L-Phe into (S)-mandelic acid; SMDH and AT to convert (S)-mandelic acid into L-Phg; an 
bioRxiv preprint doi: https://doi.org/10.1101/2022.01.14.476296; this version posted January 14, 2022. The copyright holder for this preprint (which was not certified by peer review) is the author/funder. All rights reserved. No reuse allowed without permission.

additional plasmid expressing GluDH from E. coli ${ }^{[12,18]}$ to improve the L-glutamate regeneration. When the recombinant $E$. coli strain Ec-Phg1.0 harboring three plasmids ( $\mathrm{PET}$-LAADHmaS, pRSF-SMDH-AT and pACYC-GluDH) was analyzed by the SDS-PAGE, we could clearly observe the bands corresponding to HmaS, SMDH, AT and GluDH (Figure 1b). However, LAAD was not observed from the SDS-PAGE result, probably because LAAD is a membrane-associated protein

(a)

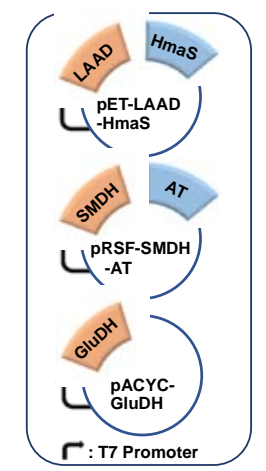

(b)

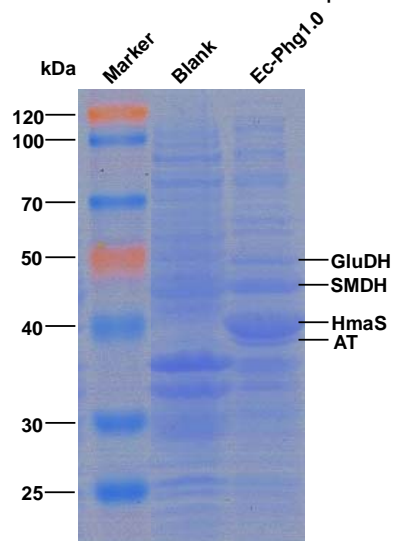

which is less abundant.

Figure 1. Plasmid design of aminotransferase (AT)-dependent route and SDS-PAGE analysis of protein expression. (a) The recombinant $E$. coli strain Ec-Phg1.0 containing three plasmids (pET-LAAD-HmaS, pRSF-SMDHAT and pACYC-GluDH). (b) SDS-PAGE analysis of the recombinant $E$. coli strain Ec-Phg1.0. Blank: E. coli BL21 (DE3) harboring empty plasmids.

In the early experiments, we compared the effect of different $\mathrm{pH}$ conditions on L-Phg production, and we found that the ideal $\mathrm{pH}$ for L-Phg synthesis was around 8.0 (Figure 2a). As shown in Figure $2 \mathrm{~b}$ and Table S1, when $10 \mathrm{mM}$ L-Phe was fed to the recombinant $E$. coli Ec-Phg1.0 expressing five enzymes, 7.21 \pm $0.15 \mathrm{mM}$ L-Phg was obtained after $48 \mathrm{~h}$, which corresponds to $\sim 72.1 \%$ conversion. From the HPLC result, we found a

(a)

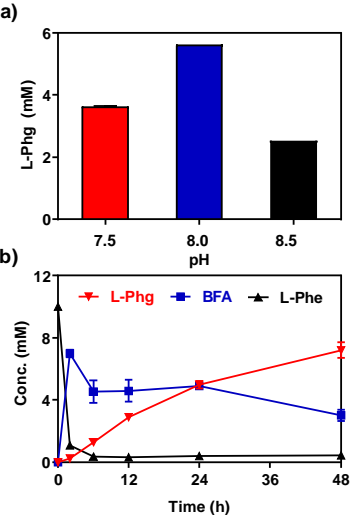

(c)

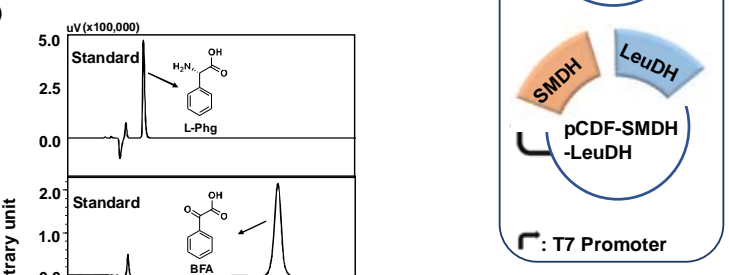

Data represent the mean value with standard deviations from triplicate of experiments.

According to the literature, LAAD from $P$. mirabilis showed a broad substrate activity on L-amino acids, such as L-His, L-Arg, L-Phe and so on ${ }^{[19]}$. We hypothesized that LAAD might deaminate L-Phg, which leads to a futile catalytic cycle and results in the accumulation of BFA. To corroborate this hypothesis, we further carried out the L-Phg stability test. When $10 \mathrm{mM}$ L-Phg was treated with the recombinant $E$. coli expressing LAAD, no appreciable degradation of L-Phg was observed even after $48 \mathrm{~h}$ (Supplementary Figure S1). Therefore, we suspected that the transamination step catalyzed by AT/GluDH might be the bottleneck limiting the L-Phg production. As it was reported that the production of $34 \mathrm{mM} \mathrm{L-Phg} \mathrm{from} 40$ $\mathrm{mM}$ L-Phe was achieved in $E$. coli containing eight reaction steps with AT/GluDH and there was no accumulation of BFA in the experimental process ${ }^{[12]}$, the AT activity is unlikely to be ratelimiting. However, LAAD from $P$. mirabilis also effectively deaminates $L$-glutamate to $a$-ketoglutarate ${ }^{[20]}$. Thus, we inferred that the amine donor of L-glutamate was degraded by LAAD, thereby leading to the accumulation of BFA and poor yield of $L$ Phg.

To overcome the low catalytic efficiency of AT/GluDH-mediated route toward L-Phg production, we next sought to find an alternative strategy to skip the use of amine donor of Lglutamate. From the literature, the preparation of L-Phg in E. coli could be realized by expressing an amino acid dehydrogenase from Bacillus clausii (BcAADH) with optimal $\mathrm{pH}$ around 9.5-10.5 [21], LeuDH from Exiguobacterium sibiricum with optimal $\mathrm{pH}$ around $10.0,{ }^{[22]}$ and LeuDH from $B$. cereus with optimal $\mathrm{pH}$ around $8.0^{[16]}$. In this study, we decided to replace AT/GluDH with LeuDH from $B$. cereus, which is more compatible with our establishment reaction condition (Scheme 1). As shown in Figure $3 a$, we further constructed the recombinant $E$. coli strain (a)

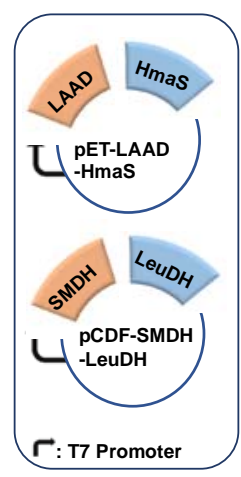

(b)

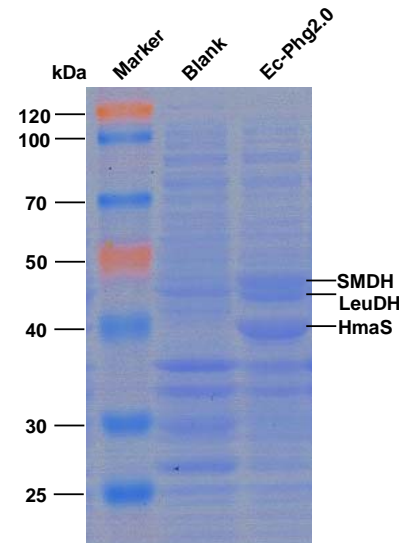

Ec-Phg2.0 containing two plasmids (pET-LAAD-HmaS and pRSF-SMDH-LeuDH). From the SDS-PAGE analysis, we could observe the bands corresponding to HmaS, SMDH and LeuDH in the recombinant E. coli strain Ec-Phg2.0 upon the IPTG induction (Figure 3b).

Figure 3. Plasmid design of leucine dehydrogenase (LeuDH)-dependent route and SDS-PAGE analysis of protein expression. (a) The recombinant E. coli strain Ec-Phg2.0 containing two plasmids (pET-LAAD-HmaS and pRSF-SMDH-LeuDH). (b) SDS-PAGE analysis of the recombinant $E$. coli strain Ec-Phg2.0. Blank: E. coli BL21 (DE3) harboring empty plasmids.

As can be seen from Figure $4 \mathrm{a}$ and Table S2, when $10 \mathrm{mM} \mathrm{L-}$ Phe was used as the substrate, $9.92 \pm 0.39 \mathrm{mM}$ L-Phg was 
bioRxiv preprint doi: https://doi.org/10.1101/2022.01.14.476296; this version posted January 14, 2022. The copyright holder for this preprint (which was not certified by peer review) is the author/funder. All rights reserved. No reuse allowed without permission.

produced after $12 \mathrm{~h}$. Interestingly, the problem of BFA accumulation was addressed by replacing AT/GluDH module with LeuDH from $B$. cereus (Figure 4c). To compare the catalytic efficiency of our system with that of the recombinant $E$. coli
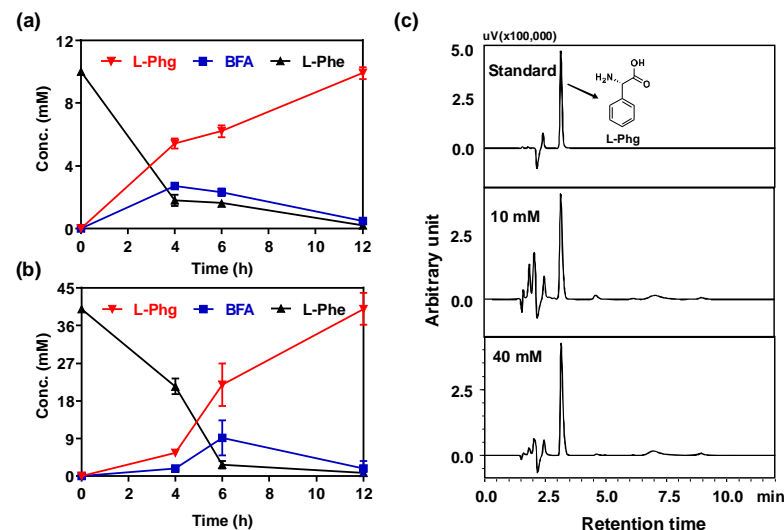

expressing 12 genes ${ }^{[12]}$, we also examined high substrate concentration of $40 \mathrm{mM} \mathrm{L-Phe.} \mathrm{When} 40 \mathrm{mM} \mathrm{L-Phe} \mathrm{was} \mathrm{fed} \mathrm{to}$ the recombinant $E$. coli, approximately $39.97 \pm 3.84 \mathrm{mM}(6.04 \pm$ $0.58 \mathrm{~g} / \mathrm{L}$ ) L-Phg was obtained after $12 \mathrm{~h}$ (Figure 4b, Table S3), which corresponds to $>99.9 \%$ conversion.

Figure 4. Characterization of LeuDH-dependent route for L-Phg synthesis. (a) Time course of biotransforming L-Phe $(10 \mathrm{mM})$ to L-Phg. (b) Time course of bioconverting L-Phe $(40 \mathrm{mM})$ to L-Phg. (c) Representative HPLC result showing L-Phg produced by the recombinant E. coli strain Ec-Phg2.0. All experiments mentioned above were carried out in $200 \mathrm{mM} \mathrm{KP}$ buffer with $10 \mathrm{~g}$ cdw $\mathrm{L}^{-1}$ recombinant $E$. coli at $30^{\circ} \mathrm{C}$. Data represent the mean value with standard deviations from triplicate of experiments.

In summary, we have developed two artificial enzymatic cascades to synthesize enantiopure amino acid of L-Phg from biobased L-Phe. The first enzymatic cascade comprises coexpressing five enzymes in the recombinant E. coli strain EcPhg1.0 harboring three corresponding plasmids ( $p E T-L A A D-$ HmaS, pRSF-SMDH-AT and PACYC-GluDH). However, under the optimal $\mathrm{pH}$ condition, only $7.21 \pm 0.15 \mathrm{mM}$ L-Phg was obtained from $10 \mathrm{mM} \mathrm{L-Phe} \mathrm{after} 48 \mathrm{~h}$ and the accumulation of intermediate product BFA was observed. Further experiments revealed that $L A A D$ from $P$. mirabilis could not deaminate $L-P h g$ (Supplementary Figure $\mathrm{S} 1$ ), indicating that the transamination step is rate-limiting. Next, we attempted to use LeuDH from $B$. cereus to replace $A T / G l u D H$ and the recombinant $E$. coli strain Ec-Phg2.0 harboring two corresponding plasmids ( $p E T$-LAAD$\mathrm{HmaS}$ and pRSF-SMDH-LeuDH) was constructed. The four-step enzymatic cascade could efficiently convert $40 \mathrm{mM}$ of L-Phe to $39.97 \pm 3.84 \mathrm{mM}(6.04 \pm 0.58 \mathrm{~g} / \mathrm{L})$ L-Phg, reaching $>99.9 \%$ conversion after $12 \mathrm{~h}$. Although we did not further optimize and scale-up the biocatalytic process, it would be possible to achieve even higher L-Phg titer as more active enzyme alternatives are available for L-Phg synthesis from mandelate ${ }^{[21-22,25]}$. Based on these findings, the four-step enzymatic cascade outperformed all the previously established biocatalytic systems for L-Phg synthesis from biobased L-Phe ${ }^{[12,24]}$. Since there was no appreciable accumulation of intermediates for E. coli strain EcPhg2.0, our biocatalytic method would greatly simplify the product separation process, with a great potential for future industrial applications.

\section{Acknowledgements}

This work was supported by Xiamen University under grant no. 0660-X2123310 and ZhenSheng Biotech, China.

Keywords: L-phenylglycine; L-amino acid deaminase; multienzyme cascade; leucine dehydrogenase; whole-cell biocatalyst.

[1] a) G. Helmchen, A. Pfaltz, Acc Chem. Res. 2000, 33, 336345; b) M. Breuer, K. Ditrich, T. Habicher, B. Hauer, M. Kesseler, R. Sturmer, T. Zelinski, Angew. Chem. Int. Ed. 2004, 43, 788-824.

[2] L. M. Van Langen, F. Van Rantwijk, V. K. Švedas, R. A. Sheldon, Tetrahedron Asymmetry. 2000, 11, 1077-1083.

a) R. S. Al Toma, C. Brieke, M. J. Cryle, R. D. Süssmuth, Nat. Prod. Rep. 2015, 32, 1207-1235; b) L. J. MarcosZambrano, P. Escribano, E. Bouza, J. Guinea, Int. J. Med. Microbiol. 2014, 304, 1192-1198.

[4] Y. J. Mast, W. Wohlleben, E. Schinko, J. Biotechnol. 2011, 155, 63-67.

[5] C. Cocito, Microbiol. Rev. 1979, 43, 145-192.

[6] J. Wang, X. Liu, X. Feng, Chem Rev 2011, 111, 69476983.

[7] a) S. Aiba, N. Takamatsu, T. Sasai, Y. Tokunaga, T. Kawasaki, Chem. Commun. (Camb) 2016, 52, 1083410837; b) S. Miyagawa, K. Yoshimura, Y. Yamazaki, N. Takamatsu, T. Kuraishi, S. Aiba, Y. Tokunaga, T. Kawasaki, Angew. Chem. Int. Ed. 2017, 56, 1055-1058; c) I. Baglai, M. Leeman, K. Wurst, B. Kaptein, R. M. Kellogg, W. L. Noorduin, Chem. Commun. 2018, 54, 10832-10834.

[8] a) S. Saravanan, A. Sadhukhan, N. U. Khan, R. I. Kureshy, S. H. Abdi, H. C. Bajaj, J. Org. Chem. 2012, 77, 43754384; b) W. H. Boesten, J. P. Seerden, B. de Lange, H. J. Dielemans, H. L. Elsenberg, B. Kaptein, H. M. Moody, R. M. Kellogg, Q. B. Broxterman, Org. Lett. 2001, 3, 11211124.

[9] J. Muschiol, C. Peters, N. Oberleitner, M. D. Mihovilovic, U. T. Bornscheuer, F. Rudroff, Chem. Commun. 2015, 51, $5798-5811$.

[10] a) J.-B. Wang, M. T. Reetz, Nat. Chem. 2015, 7, 948-949; b) S. Servi, D. Tessaro, G. Pedrocchi-Fantoni, Coordin. Chem. Rev. 2008, 252, 715-726.

[11] a) W. Leuchtenberger, K. Huthmacher, K. Drauz, Appl. Microbiol. Biotechnol. 2005, 69, 1-8; b) A. Rodriguez, J. A. Martnez, N. Flores, A. Escalante, G. Gosset, F. Bolivar, Microb. Cell Fact. 2014, 13, 126-141.

[12] Y. Zhou, S. Wu, Z. Li, Angew. Chem. Int. Ed. 2016, 128, 11819-11822.

[13] a) G. Massad, H. Zhao, H. L. Mobley, J. Bacteriol. 1995, 177, 5878-5883; b) Y. Hou, G. S. Hossain, J. Li, H. D. Shin, L. Liu, G. Du, Appl. Microbiol. Biotechnol. 2015, 99, 8391-8402.

[14] U. Muller, F. van Assema, M. Gunsior, S. Orf, S. Kremer, D. Schipper, A. Wagemans, C. A. Townsend, T. Sonke, R. Bovenberg, M. Wubbolts, Metab. Eng. 2006, 8, 196-208.

[15] B. Mitra, J. A. Gerlt, P. C. Babbitt, C. W. Koo, G. L. Kenyon, D. Joseph, G. A. Petsko, Biochemistry. 1993, 32, 12959-12967.

[16] Q. Liu, J. Zhou, T. Yang, X. Zhang, M. Xu, Z. Rao, Appl. Microbiol. Biotechnol. 2018, 102, 2129-2141.

[17] a) L. Liu, Y. Zhu, Y. Chen, H. Chen, C. Fan, Q. Mo, J. Yuan, Chem. Asian J. 2020, 15, 1018-1021; b) Y. Zhu, T. Yang, Y. Chen, C. Fan, J. Yuan, ChemistrySelect. 2020, 5, 14292-14295. 
bioRxiv preprint doi: https://doi.org/10.1101/2022.01.14.476296; this version posted January 14, 2022. The copyright holder for this preprint (which was not certified by peer review) is the author/funder. All rights reserved. No reuse allowed without permission.

[18] S. Wu, Y. Zhou, T. Wang, H.-P. Too, D. I. C. Wang, Z. Li, Nat. Commun. 2016, 7, 11917.

[19] G. Molla, R. Melis, L. Pollegioni, Biotechnol. Adv. 2017, 35, 657-668.

[20] J. O. Baek, J. W. Seo, O. Kwon, S. I. Seong, I. H. Kim, C. H. Kim, J. Basic Microbiol. 2011, 51, 129-135.

[21] J. Cheng, G. Xu, R. Han, J. Dong, Y. Ni, RSC Adv. 2016, 6, 80557-80563.
[22] C.-W. Fan, G.-C. Xu, B.-D. Ma, Y.-P. Bai, J. Zhang, J.-H. Xu, J. Biotechnol. 2015, 195, 67-71.

[23] S. P. Liu, R. X. Liu, A. A. El-Rotail, Z. Y. Ding, Z. H. Gu, L. Zhang, G. Y. Shi, J. Biotechnol. 2014, 186, 91-97.

S. P. Liu, R. X. Liu, J. Mao, L. Zhang, Z. Y. Ding, Z. H. Gu, G. Y. Shi, Biotechnol. Bioproc. E. 2016, 21, 153-159.

[25] V. Resch, W. M. F. Fabian, W. Kroutil, Adv. Synth. Catal. 2010, 352, 993-997. 


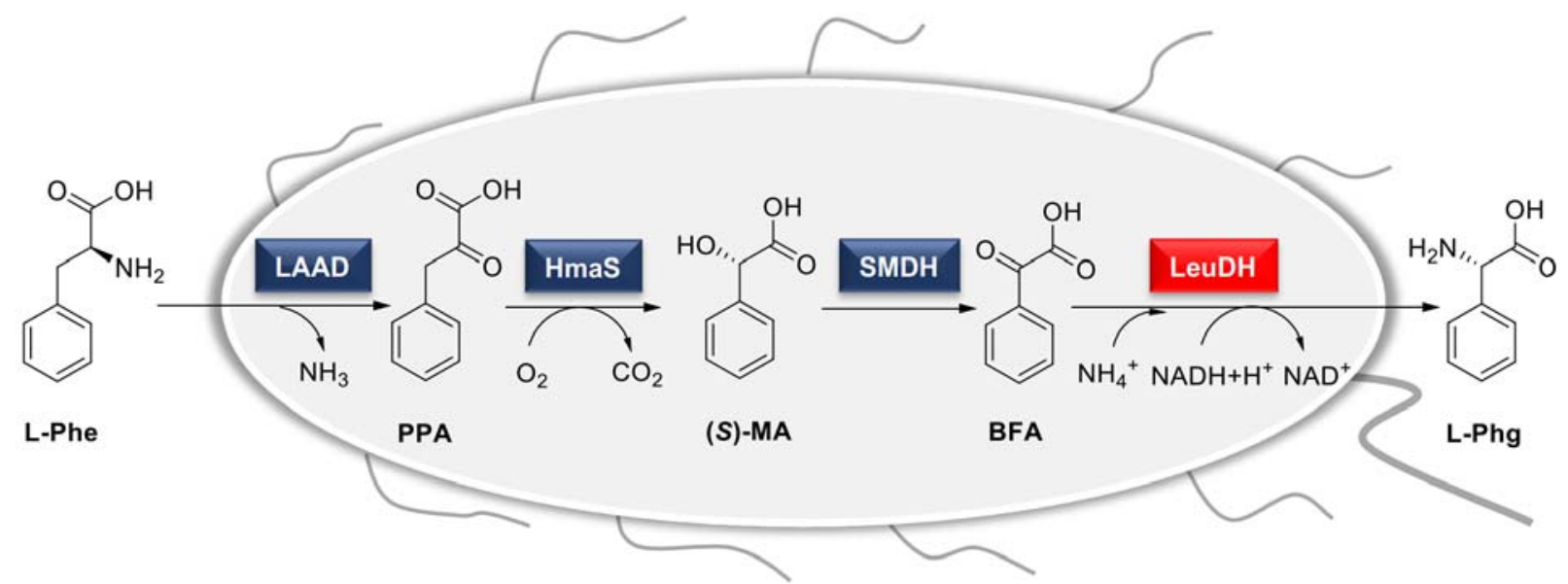

Graphical abstract: a concise four-step enzymatic cascade for the L-phenylglycine synthesis from biobased L-phenylalanine was devised. $40 \mathrm{mM}$ L-phenylalanine afforded the synthesis of $39.97 \pm 3.84 \mathrm{mM}(6.04 \pm 0.58 \mathrm{~g} / \mathrm{L})$ L-phenylglycine, reaching $99.9 \%$ conversion 